\title{
Interplay between real and pseudomagnetic field in graphene with strain
}

\author{
Kyung-Joong Kim, ${ }^{1}$ Ya. M. Blanter, ${ }^{2}$ and Kang-Hun $\mathrm{Ahn}{ }^{1,3,}{ }^{*}$ \\ ${ }^{1}$ Department of Physics, Chungnam National University, Daejeon 305-764, Republic of Korea \\ ${ }^{2}$ Kavli Institute of Nanoscience, Delft University of Technology, Lorentzweg 1, NL-2628 CJ Delft, The Netherlands \\ ${ }^{3}$ Department of Physics, University of Bath, Bath BA2 7AY, United Kingdom
}

(Received 13 July 2011; published 5 August 2011)

\begin{abstract}
We investigate electric and magnetic properties of graphene with rotationally symmetric strain. The strain generates a large pseudomagnetic field with alternating sign in space, which forms a strongly confined quantum dot connected to six chiral channels. The orbital magnetism, degeneracy, and channel opening can be understood from the interplay between the real and pseudomagnetic fields which have different parities under time reversal and mirror reflection. While the orbital magnetic response of the confined state is diamagnetic, it can be paramagnetic if there is an accidental degeneracy with opposite mirror reflection parity.
\end{abstract}

DOI: 10.1103/PhysRevB.84.081401

PACS number(s): 73.22.Pr, 71.23.-k, 71.55.Jv, 75.30.Cr

The recent successful preparation of a one-atom layer of carbons, graphene, ${ }^{1,2}$ has provided the opportunity for theoretical and experimental research of a massless Dirac fermion in nanoelectronics. While quantum dots which confine quasiparticles in graphene are basic building blocks for its nanoelectronic application, the confinement turns out to be nontrivial. It is because, in graphene, where the quasiparticles are described by massless Dirac fermions, they can penetrate large and wide electrostatic barriers due to the effect of Klein tunneling. ${ }^{3}$ In principle, graphene dots can be realized by a spatially inhomogeneous magnetic field, but the required magnetic field for the confinement, however, is unreasonably strong ${ }^{4}$ compared to usual electronics applications. Recently, strain engineering of graphen ${ }^{5-7}$ has attracted great attention as an alternative tool for graphene electronics because the strain induces a strong pseudomagnetic field which guides electrons. Thus, for the strained graphene to work successfully in combination with existing technologies, it is now important to understand the physical properties of the pseudomagnetic field. In this Rapid Communication, we investigate the relative contribution of real and pseudomagnetic fields to the electric and magnetic properties of the graphene. We show that a reasonable size of strain can generate a strong pseudomagnetic field to form a graphene quantum dot with six chiral channels. It will be demonstrated that the different symmetry of real and pseudomagnetic fields give rises to rich properties of channel opening and orbital magnetism. The pseudomagnetic field appears since the variation of hopping energies by elastic strains enters the Dirac equation..$^{8-13}$ While the strong confinement is due to the fact that the pseudomagnetic field is very strong $(\sim 10 \mathrm{~T})$, the six chiral channels are due to the topology of the pseudomagnetic field, where charged particles propagate along the zero-field line. As we will show here, the real and pseudomagnetic fields have different parities under the symmetry operation, such as time reversal and mirror reflection. From the symmetry arguments, we prove that while the real magnetic field breaks the time-reversal symmetry in its Hamiltonian, it does not lift the valley degeneracy. We will demonstrate our theory by showing orbital diamagnetism of the confined state. It will be shown that a paramagnetic response is also allowed when a partially open state with opposite parity becomes degenerate with the confined state.
Let us consider graphene where mechanical deformation is allowed in a restricted disk shape. This can be realized by a circular hole made in the substrate below the graphene sheet, and the deformation is induced through an external force. In experiments, circularly symmetric strain fields can be applied with an atomic force microscopy (AFM) tip or by a homogeneous gas pressure acting on graphene below the substrate. ${ }^{14}$ When strain is induced by a homogeneous load, the optimized vertical displacement $h(\mathbf{r})$ is given by ${ }^{15}$

$$
h(\mathbf{r})=\frac{f_{0}}{4^{3} D}\left[R^{2}-\left(x^{2}+y^{2}\right)\right]^{2},
$$

where $f_{0}$ is the force per unit area acting on the surface, $D$ is the bending rigidity, $h_{0}=f_{0} R^{4} /\left(4^{3} D\right)$ is the vertical displacement at the center, and $R$ is the radius of the region where the deformation is allowed. The in-plane relaxation of the carbon atoms $u_{x}, u_{y}$ can be calculated by minimizing the elastic free energy for the given vertical displacement $h(\mathbf{r})$,

$F=\int d x d y\left[\frac{\kappa}{2}\left(\nabla^{2} h\right)^{2}+\frac{\lambda}{2}\left(\sum_{i} u_{i i}\right)^{2}+\mu \sum_{i j}\left(u_{i j}\right)^{2}\right]$,

where $\kappa$ is the bending rigidity, $\lambda \approx 1 \mathrm{eV} / \AA^{2}$, and $\mu(\approx 3 \lambda)$ are Lamé coefficients, ${ }^{16}$ and $u_{i j}$ is the strain tensor. Here, the strain tensor $u_{i j}(\mathbf{r})$ is related to the displacement fields via $u_{x x}=\partial u_{x} / \partial x+\frac{1}{2}(\partial h / \partial x)^{2}, u_{y y}=$ $\partial u_{y} / \partial y+\frac{1}{2}(\partial h / \partial y)^{2}, \quad$ and $\quad u_{x y}=\frac{1}{2}\left(\partial u_{x} / \partial y+\partial u_{y} / \partial x\right)+$ $\frac{1}{2}(\partial h / \partial x)(\partial h / \partial y)$. Our numerical calculations show that the in-plane relaxation has only a radial component which can be maximally $6 \AA$. We find the in-plane relaxation weakens the pseudomagnetic field but does not affect its symmetries, which will be discussed in this Rapid Communication.

We consider spinless fermions in a graphene lattice. The spinless quasiparticle in the graphene can be described by a four-component wave function $\Psi^{\mathrm{T}}=\left(\Psi_{K}^{T}, \Psi_{-K}^{T}\right)=$ $\left(\psi_{A, K}, \psi_{B, K}, \psi_{A,-K}, \psi_{B,-K}\right)$. These are the electron wave functions near two inequivalent points (also called valleys) $\pm K$ in a hexagonal Brillouin zone in the two crystalline sublattices $A$ and $B$. We ignore the valley mixing by the strain based on the results of a tight-binding calculation showing that the two inequivalent valleys are not coupled 
(a)
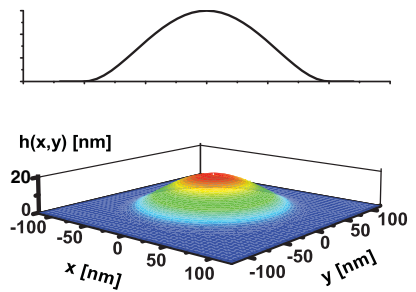

FIG. 1. (Color online) The vertical displacement of our considered systems in the text (a) and the pseudomagnetic fields (b). The radius is $R=100 \mathrm{~nm}$ and the height is $h_{0}=20 \mathrm{~nm}$.

under uniaxial deformations up to $20 \% .^{17}$ The main effect of the strain field on the electrons is to modify the energy for electron hopping between the nearest-neighbor atoms. The modified quasiparticle energy by the strain is well described by introducing the pseudogauge field $\mathbf{A}_{\mathrm{ps}}(\mathbf{r})$ in the massless Dirac equation

$$
H=v_{F}\left(\begin{array}{cc}
\vec{\sigma} \cdot\left(\mathbf{p}+e \mathbf{A}_{\mathrm{ps}}\right) & 0 \\
0 & -\vec{\sigma}^{*} \cdot\left(\mathbf{p}-e \mathbf{A}_{\mathrm{ps}}\right)
\end{array}\right),
$$

where $-e$ is the electric charge, $\mathbf{p}=-i \hbar\left(\partial_{x}, \partial_{y}\right)$, and $\vec{\sigma}=$ $\left(\sigma_{x}, \sigma_{y}\right)$ are Pauli matrices acting in the sublattice space. The Fermi velocity is given by $v_{F}=3 \mathrm{ta} / 2 \hbar$, where $t \approx 2.8 \mathrm{eV}$ is the electron hopping energy between the nearest $\pi$ orbitals and $a$ is the nearest distance between carbon atoms. Here, we choose the $x$ axis which has a zigzag structure in real space and take two $K$ points $\left[K=\frac{\pi}{a}\left(\frac{4}{3 \sqrt{3}}, 0\right),-K\right]$. The pseudogauge field $\mathbf{A}_{\mathrm{ps}}(\mathbf{r})$ is written as ${ }^{10}$

$$
\mathbf{A}_{\mathrm{ps}}=\frac{t \beta}{e v_{F}}\left(u_{x x}-u_{y y},-2 u_{x y}\right),
$$

where $\beta \equiv-\frac{\partial \ln t}{\partial \ln a} \approx 2-3$ is the dimensionless coupling parameter for the lattice deformation. ${ }^{8-11,18}$ In Fig. 1, we plot the pseudomagnetic field $\mathbf{B}_{\mathrm{ps}}=B_{\mathrm{ps}} \mathbf{k}=\nabla \times \mathbf{A}_{\mathrm{ps}}$. This is the pseudomagnetic field experienced by the particle in the $K$ valley, and for the particle in the $-K$ valley, the sign of the magnetic field is opposite.

To investigate the confinement of the quasiparticles in graphene, we compute the eigenenergies of the system as the total graphene size used in the calculation $L$ increases and observe whether the energy is insensitive to the system size. The graphene in the calculation is a disk defined as $r<L$ and the graphene is deformed only in the region of $r<R$. We impose the boundary condition $\Psi_{A}(r=L)=\Psi_{B}(r=L)=0$. While the wave functions which extend over the total system are sensitive to the boundary conditions, localized states in the deformed region are not affected by the boundary condition. We obtain the eigenvalues of the Hamiltonian using the basis functions $\psi_{A, B}=\sum c_{n, l}^{A, B} \phi_{n, l}(\mathbf{r})$, where $\phi_{n, l}(\mathbf{r})=J_{l}\left(\frac{\alpha_{n l}}{L} r\right) e^{i l \theta}$. Here, $\alpha_{n l}$ is the $n$th zero of the Bessel function $J_{l}(x)$ of order $l$, and we use the indices $l=0, \pm 1, \pm 2, \ldots, n=1,2,3, \ldots$.

As shown in Fig. 2, we find that at certain energies there exist eigenstates whose eigenenergies are insensitive to the system size $L$. These particular energy values are determined by the strength of the pseudomagnetic field. These are the

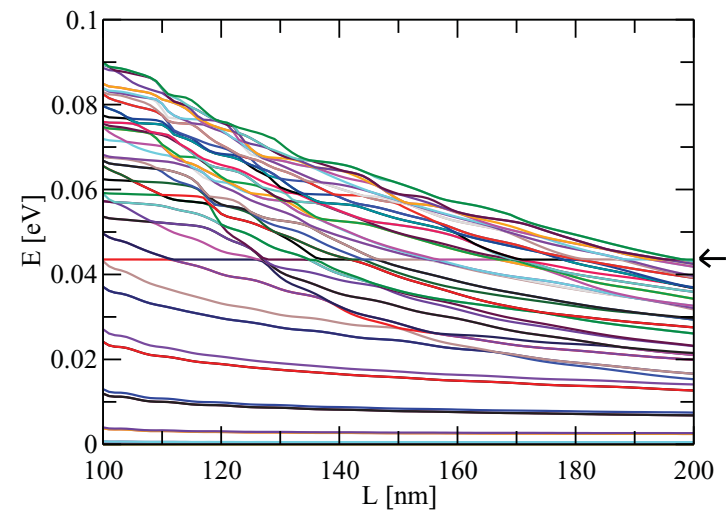

FIG. 2. (Color online) The eigenenergies as a function of the system size $L$. We set $R=100 \mathrm{~nm}$ and $h_{0}=20 \mathrm{~nm}$. Note that at a certain energy (marked by an arrow), the eigenenergy becomes insensitive to the system size $L$.

localized states induced by the deformation of the graphene. Compared to the midgap states in a ripple array studied in Ref. 8, which shows weak size dependence in a logarithmic scale, the eigenenergies of the localized states show almost no size dependence. From an analytic calculation, the length and energy scales for the localized state can be obtained by bringing the asymptotics of the differential equation for the wave function to dimensionless form. This is done by rescaling $r \rightarrow r_{0} \tilde{r}, E \rightarrow E_{0} \tilde{E}$, with

$$
r_{0}=\left(\frac{\hbar v_{F} R^{4}}{8 t \beta h_{0}^{2}}\right)^{1 / 3}, \quad E_{0}=\hbar v_{F} / r_{0} .
$$

The scale $r_{0}$ thus plays a role in the localization length and can be estimated as $\left(R^{4} a / h_{0}^{2}\right)^{1 / 3}$, where $a$ is the lattice constant. Since the localization length must be shorter than the hole radius, otherwise the Dirac equation with a pseudomagnetic field cannot apply, the localization length expressed in the above equation is valid only for a strong enough load $h_{0} \gg$ $\sqrt{R a}$. The scale $E_{0}$ is associated with the depth of the potential well and the energy of the localized energy levels.

It proves useful to consider the symmetries to understand the energy spectra of quantum systems. The time-reversal symmetry is not broken by the strain if we consider the problem with both of the valleys. A time-reversal operation defined by

$$
\mathcal{T}=\tau_{x} \mathcal{K}
$$

satisfies $\mathcal{T} H \mathcal{T}^{-1}=H, \mathcal{T} i \mathcal{T}^{-1}=-i .{ }^{19}$ Here $\mathcal{K}$ is the complex conjugate operator and $\left(\tau_{x}, \tau_{y}, \tau_{z}\right)$ are the Pauli matrices acting in the valley space. Note that the Kramers degeneracy is not relevant here since the original system has an orthogonal symmetry $\mathcal{T}^{2}=1$.

The quasiparticle can remain in a given valley provided there is no short-range scattering (e.g., lattice defects). In this restricted case of a single valley, the time-reversal symmetry is broken by the pseudomagnetic field. The usual two-component time-reversal operator for $1 / 2 \operatorname{spin} \mathcal{S}=-i \sigma_{y} \mathcal{K}$ does not commute with the Hamiltonian in Eq. (3). The Hamiltonian is symmetric under the symplectic time-reversal transformation only in the absence of the strain $\left(\mathcal{S}^{2}=-1\right) ; \mathcal{S} H\left(\mathbf{A}_{\mathrm{ps}}=\right.$ $0) \mathcal{S}^{-1}=-H\left(\mathbf{A}_{\mathrm{ps}}=0\right)$. The relevant Kramers degeneracy here is lifted by the pseudomagnetic field $\mathbf{A}_{\mathrm{ps}} \neq 0$. 

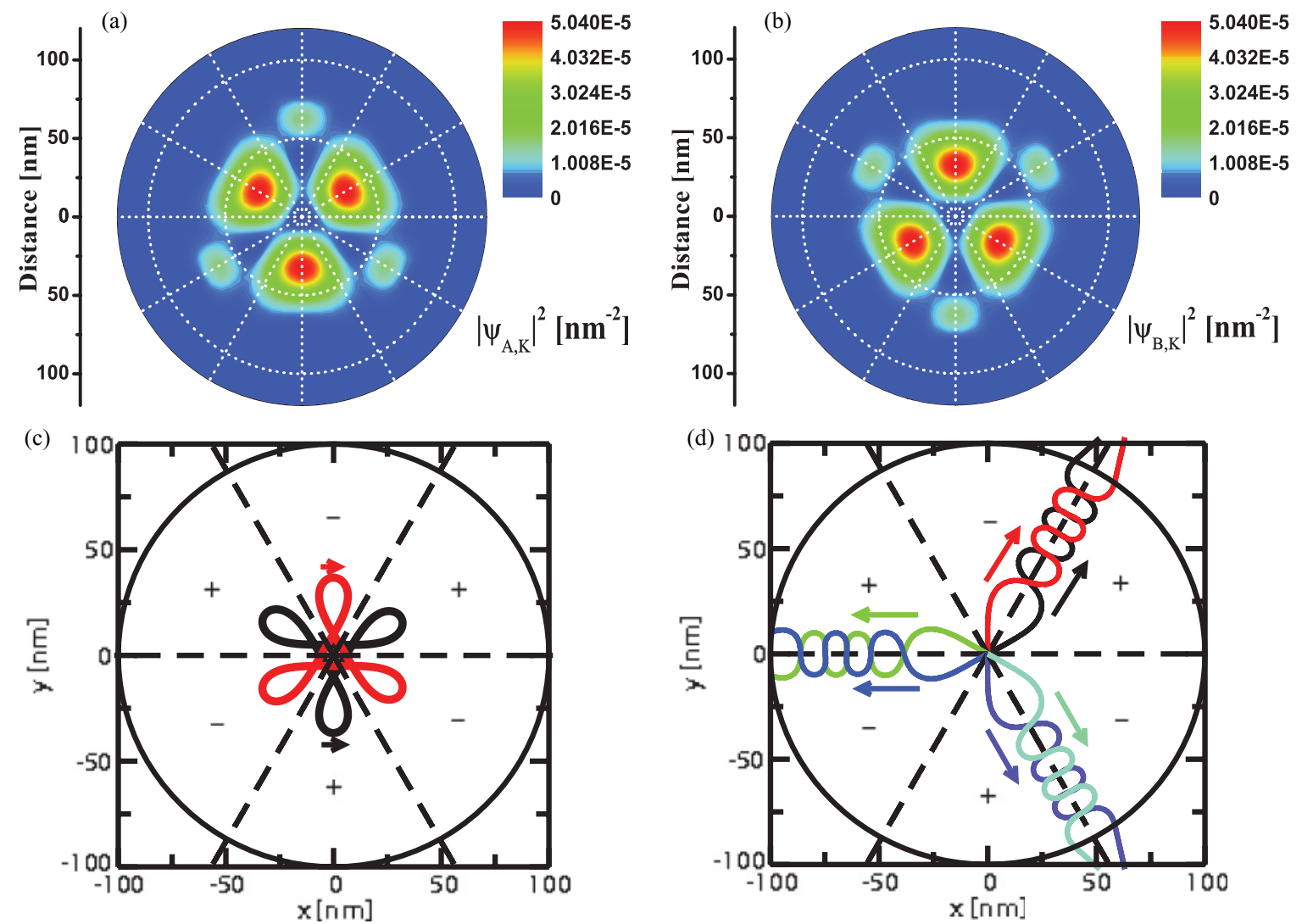

FIG. 3. (Color online) (a) Probability densities of the localized wave functions $\left|\psi_{A, K}(\mathbf{r})\right|^{2}$ and (b) $\left|\psi_{B, K}(\mathbf{r})\right|^{2}$. We set $R=100 \mathrm{~nm}, h_{0}=$ $20 \mathrm{~nm}$. (c) Classical closed orbits of a charged particle in the inhomogeneous magnetic field given by $B_{\mathrm{ps}}$ which resemble the probability density of the localized states shown in (a) and (b). (d) Classical paths describing three outgoing channels.

The Hamiltonian in Eq. (3) is also symmetric under a mirror reflection

$$
\begin{gathered}
\mathcal{M}=\sigma_{x} \pi_{x}, \\
\mathcal{M} H \mathcal{M}^{-1}=H,
\end{gathered}
$$

where $\pi_{x}$ acts as $x \rightarrow x, y \rightarrow-y$. The valley index remains the same under the mirror reflection but inevitably changes its lattice index. The spatial symmetry $\left(\left|\psi_{A, K}(x, y)\right|^{2}=\right.$ $\left.\left|\psi_{B, K}(x,-y)\right|^{2}\right)$ of the probability density of the localized state shown in Figs. 3(a) and 3(b) reflects the $\mathcal{M}$ symmetry in the Hamiltonian.

To understand the electronic structure of the strained graphene, we rewrite the Hamiltonian in Eq. (3) for a given valley $K$ and eigenenergy $E$,

$$
\left[v_{F}^{2}\left(\mathbf{p}+e \mathbf{A}_{\mathrm{ps}}\right)^{2}+\hbar e v_{F}^{2}\left(\nabla \times \mathbf{A}_{\mathrm{ps}}\right)_{z} \sigma_{z}\right] \Psi_{K}=E^{2} \Psi_{K} .
$$

The first term on the left-hand side of Eq. (9) comes from the kinetic energy and the second term is due to the pseudo Zeeman coupling. The pseudomagnetic field is strongest at six points, forming a hexagon (Fig. 1) where local Landau levels might be formed. For a given pseudospin and valley, one can see the maximum probability density around only three points [Figs. 3(a) and 3(b)]. This is because of a necessary condition for the stable confinement on the pseudo Zeeman coupling,

$$
\left(\nabla \times \mathbf{A}_{\mathrm{ps}}\right)_{z} \sigma_{z}<0 .
$$

In this case, more energy is expended for a quasiparticle to go out to the weaker field region. The triangular (instead of hexagonal) shape of the wave function for a given lattice and valley is due to the selective stabilization by the pseudo Zeeman coupling.

The confinement of channeling in the strained graphene can be visualized by investigating classical trajectories of the charged particles in the pseudomagnetic field [Figs. 3(c) and 3(d)]. Among the periodic orbits around the pseudomagnetic field maxima, we find clover-shaped orbits which resemble the localized wave functions in Fig. 3(c). These closed orbits are very unstable against small perturbation. In quantum mechanics, the clover-shape motion might be responsible for a quantum transition between the sites of the local density maxima. We also find outgoing trajectories [Fig. 3(d)] for different initial velocities. A charged particle can propagate along the line where the magnetic field changes sign, which is the so-called snake orbit. ${ }^{20}$ Due to the symmetry of the pseudomagnetic field, there are incoming trajectories in $60^{\circ}$ rotated angles from those of the outgoing trajectories. Quantum mechanically, for given components of the lattice and valley, the graphene quantum dot is connected to three incoming and three outgoing chiral channels.

The opening of the channels manifests in the energy spectra. The localized energy level undergoes crossing and avoided crossing as the graphene size $L$ changes. [See Figs. 2 and 4(a) 

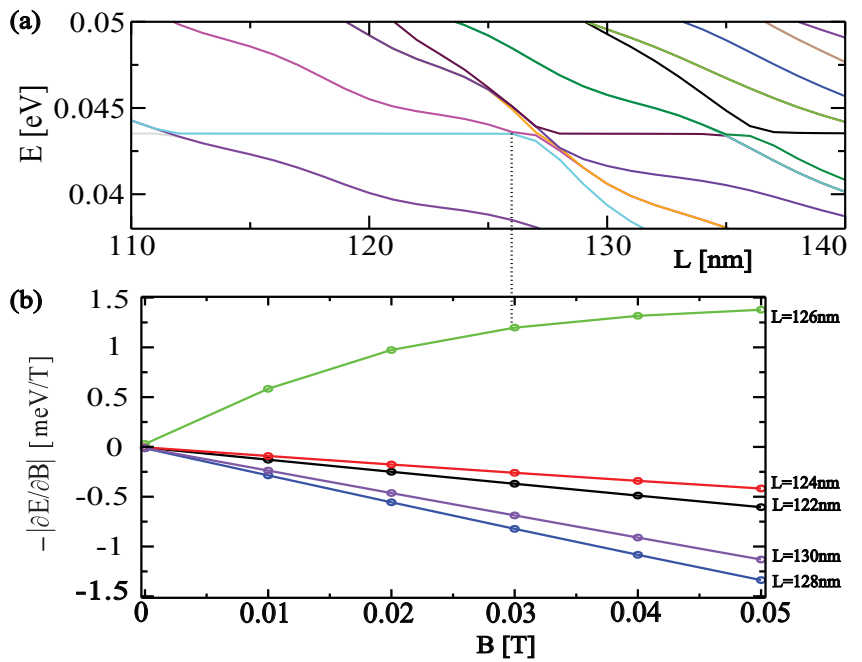

FIG. 4. (Color online) (a) A detailed energy spectra as a function of the system size $L$ which shows crossing and avoided crossing. (b) The orbital magnetic response $-\frac{\partial E}{\partial B}$ of localized states. Mostly the magnetic response is diamagnetic $-\frac{\partial E}{\partial B}<0$ but the response for a level crossing point (indicated by the dotted line) shows a paramagnetic response (positive value). We set $R=100 \mathrm{~nm}$ and $h_{0}=20 \mathrm{~nm}$.

for more details.] When the channels open, the eigenenergy of the confined state is affected by the graphene size, so it has avoided crossing. Meanwhile when the two levels cross each other, the channels remain closed and the eigenenergy of the localized state is insensitive the graphene size. In the process of avoided crossing, the confined state undergoes a transition to an outer state and a new outer state becomes localized. Since any parity does not change in this continuous process, the confined state transits only to the state with the same parity.

Let us consider the response of the strained graphene to the real magnetic field. When the real magnetic field $\mathbf{B}_{\mathrm{re}}=$ $\nabla \times \mathbf{A}_{\mathrm{re}}$ is applied, minimal coupling of the electromagnetic gauge field $\mathbf{A}_{\mathrm{re}}$ is done by replacing $\mathbf{p}$ with $\mathbf{p}+e \mathbf{A}_{\mathrm{re}}$ in Eq. (3). The Hamiltonian becomes $H+H^{\prime}$, where

$$
H^{\prime}=v_{F} e\left(\begin{array}{cc}
\vec{\sigma} & 0 \\
0 & -\vec{\sigma}^{*}
\end{array}\right) \cdot \mathbf{A}_{\mathrm{re}},
$$

and $\mathbf{A}_{\mathrm{re}}=\frac{1}{2}(-y, x) B$ is the gauge field for the real magnetic field in the $z$ direction.
We want to address here that the application of the real magnetic field breaks the time-reversal symmetry of the Hamiltonian, but it does not lift the valley degeneracy. This can be proved by showing $\left\langle\Psi_{ \pm K}\left|\frac{\partial H^{\prime}}{\partial B}\right| \Psi_{ \pm K}\right\rangle=0$. The proof comes from the fact that the eigenstates have either an even or odd parity of the mirror reflection symmetry in Eq. (8), $\mathcal{M}^{2}=1: \quad\left\langle\Psi_{K}\left|\frac{\partial H^{\prime}}{\partial B}\right| \Psi_{K}\right\rangle=\left\langle\Psi_{K}\left|\left(-y \sigma_{x}+x \sigma_{y}\right)\right| \Psi_{K}\right\rangle=$ $\left\langle\Psi_{K}\left|\mathcal{M}\left(+y \sigma_{x}-x \sigma_{y}\right) \mathcal{M}\right| \Psi_{K}\right\rangle=-\left\langle\Psi_{K}\left|\left(-y \sigma_{x}+x \sigma_{y}\right)\right| \Psi_{K}\right\rangle$. Since $\left\langle\Psi_{K}\left|\frac{\partial H^{\prime}}{\partial B}\right| \Psi_{K}\right\rangle$ is equal to its own negative value, it must be zero. The leading magnetic field dependence of the eigenenergy in the presence of the magnetic field is not linear but quadratic, $\propto B^{2}$. It comes from the kinetic energy and its sign is positive. Therefore, the orbital magnetization of the strain-induced quantum dot at zero temperature is diamagnetic $(-\partial E / \partial B<0)$ and is proportional to the applied magnetic field strength [see Fig. 4(b)].

In contrast to the diamagnetic response of the confined state, the orbital magnetic response can be paramagnetic $(-\partial E / \partial B>0)$ when there are level crossings. Near the region of the level crossings, there are two energy levels with an opposite parity of $\mathcal{M}$. One of the states is a localized state and the other is a partially opened state (not shown). The accidental degeneracy which occurred here can be lifted by applying a real magnetic field because $H^{\prime}$ is odd under the mirror reflection $\mathcal{M} H^{\prime} \mathcal{M}=-H^{\prime}$. Then energy splitting arises, proportional to the real magnetic field strength, which contributes to the paramagnetic response.

In conclusion, we have shown that the rotationally symmetric strain in graphene can be considered a quantum dot with spatially separated six chiral channels. The chiral channels exist along the line where the pseudomagnetic field changes sign. The real and pseudomagnetic fields have different symmetries under a mirror reflection, which causes the orbital magnetism to be diamagnetic or paramagnetic depending on the degeneracy. The orbital magnetic response of the confined state is diamagnetic due to its kinetic energy. When there is an degeneracy with opposite mirror reflection parity, the orbital magnetism can be paramagnetic. Quite recently, we became aware of a work on the dynamics of electrons in strain-induced pseudomagnetic fields. $^{21}$

The authors thank M. Sieber, K. Richter, Y. Son, F. Guinea, and M. Fogler for useful discussions. This work was supported by the National Research Foundation, funded by the Korea government (No. KRF-2008-C00140). K.H.A. is thankful for financial support from Chungam National University and EU.

\footnotetext{
*ahnkh@cnu.ac.kr

${ }^{1}$ K. S. Novoselov, A. K. Geim, S. V. Morozov, D. Jiang, Y. Zhang, S. V. Dubonos, I. V. Grigorieva, and A. A. Firsov, Science 306, 666 (2004); K. S. Novoselov, A. K. Geim, S. V. Morozov, D. Jiang, M. I. Katsnelson, I. V. Grigorieva, S. V. Dubonos, and A. A. Firsov, Nature (London) 438, 197 (2005).

${ }^{2}$ Y. Zhang, Y.-W. Tan, H. L. Stormer, and P. Kim, Nature (London) 438, 201 (2005).

${ }^{3}$ V. V. Cheianov and V. I. Falko, Phys. Rev. B 74, 041403 (2006); M. I. Katsnelson, K. S. Novoselov, and A. K. Geim, Nat. Phys. 2, 620 (2006).
}

${ }^{4}$ D. Wang and G. Jin, Phys. Lett. A 373, 4082 (2009).

${ }^{5}$ Vitor M. Pereira and A. H. Castro Neto, Phys. Rev. Lett. 103, 046801 (2009).

${ }^{6}$ N. Levy, S. A. Burke, K. L. Meaker, M. Panlasigui, A. Zettl, F. Guinea, A. H. Castro Neto, and M. F. Crommie, Science 329, 544 (2010).

${ }^{7}$ T. Low and F. Guinea, Nano Lett. 10, 3551 (2010).

${ }^{8}$ F. Guinea, M. I. Katsnelson, and M. A. H. Vozmediano, Phys. Rev. B 77, 075422 (2008).

${ }^{9}$ Eun-Ah Kim and A. H. Castro Neto, Europhys. Lett. 84, 57007 (2008). 
${ }^{10}$ J. L. Manẽs, Phys. Rev. B 76, 045430 (2007).

${ }^{11}$ F. Guinea, B. Horovitz, and P. Le Doussal, Phys. Rev. B 77, 205421 (2008).

${ }^{12}$ A. F. Morpurgo and F. Guinea, Phys. Rev. Lett. 97, 196804 (2006).

${ }^{13}$ F. Guinea, M. I. Katsnelson, and A. K. Geim, Nat. Phys. 6, 30 (2010).

${ }^{14}$ J. S. Bunch, S. S. Verbridge, J. S. Alden, A. M. van der Zande, J. M. Parpia, H. G. Craighead, and P. L. McEuen, Nano Lett. 8, 2458 (2008).

${ }^{15}$ L. D. Landau and E. M. Lifshitz, Theory of Elasticity (Pergamon, London, 1959).
${ }^{16}$ F. Guinea, Baruch Horovitz, and P. Le Doussal, Solid State Commun. 149, 1140 (2009).

${ }^{17}$ V. M. Pereira, A. H. Castro Neto, and N. M. R. Peres, Phys. Rev. B 80, 045401 (2009).

${ }^{18}$ H. Suzuura and T. Ando, Phys. Rev. B 65, 235412 (2002); T. Ando, J. Phys. Soc. Jpn. 75, 124701 (2006).

${ }^{19}$ J. D. Bjorken and S. D. Drell, Relativistic Quantum Mechanics (McGraw-Hill, New York, 1964), p. 72.

${ }^{20}$ H.-S. Sim, K. H. Ahn, K. J. Chang, G. Ihm, N. Kim, S. J. Lee, Phys. Rev. Lett. 80, 1501 (1998).

${ }^{21}$ G. M. M. Wakker, R. P. Tiwari, and M. Blaauboer, e-print arXiv:1105.3588. 